\title{
A NEW APPROACH TO JACOBI'S THEOREMS VIA RAMANUJAN'S CONTINUED FRACTIONS
}

\author{
Chandrashekar Adiga and Jung Hun Han
}

In this paper, we show that Jacobi's two-square and two-triangular number theorems are immediate consequence of Ramanujan's continued fraction identities.

\section{INTRODUCTION}

Let $r_{k}(n)$ denote the number of ways the positive integer $\mathrm{n}$ can be represented as a sum of $k$ squares, with representations arising from different signs and from different orders being regarded as distinct. Geometrically, $r_{k}(n)$ counts the number of lattice points on the $k$-dimensional sphere $x_{1}^{2}+\cdots+x_{k}^{2}=n$. Let

$$
\phi(q)=\sum_{n=-\infty}^{\infty} q^{n^{2}},|q|<1
$$

Then the generating function for $r_{k}(n)$ is

$$
\phi^{k}(q)=\sum_{n=0}^{\infty} r_{k}(n) q^{n} .
$$

A triangular number is a number of the form $k(k+1) / 2$ for some non-negative integer $k$. Let $t_{k}(n)$ denote the number of representations of $\mathrm{n}$ as a sum of $k$ triangular numbers. Geometrically, $2^{k} t_{k}(n)$ counts the number of lattice points on the $k$-dimensional sphere centred at $(-1 / 2, \ldots,-1 / 2)$ with radius $\sqrt{2 n+k / 4}$. Let

$$
\psi(q)=\sum_{n=0}^{\infty} q^{n(n+1) / 2},|q|<1 .
$$

Then the generating function for $t_{k}(n)$ is

$$
\psi^{k}(q)=\sum_{n=0}^{\infty} t_{k}(n) q^{n} .
$$

Received 23rd August, 2004

Copyright Clearance Centre, Inc. Serial-fee code: 0004-9727/05 \$A2.00+0.00. 
One of the main problems is to find formulae for determining $r_{k}(n)$, in terms of simple arithmetical functions such as divisor functions. Jacobi's two-square and twotriangular-number theorems state that

$$
r_{2}(n)=4\left[d_{1}(n)-d_{3}(n)\right]
$$

and

$$
t_{2}(n)=d_{1}(4 n+1)-d_{3}(4 n+1) .
$$

where $d_{i}(m)$ is the number of divisors of $m$ congruent to i modulo 4. Many authors gave proofs of (1.1) and (1.2) using $q$-series identities. Hirschhorn [8] and Ewell [6] have used Jacobi's triple product identity to obtain (1.1). Askey [3], Adiga [1], Bhargava and Adiga [4], Fine [7] and, Cooper and Lam [5] have employed Ramanujan's ${ }_{1} \psi_{1}$ summation formula to derive (1.1) and (1.2). We show that (1.1) and (1.2) are immediate consequences of two continued fraction identities of Ramanujan.

Ramanujan's general theta function and its special cases are defined as follows:

$$
\begin{aligned}
f(a, b) & :=\sum_{n=-\infty}^{\infty} a^{n(n+1) / 2} b^{n(n-1) / 2},|a b|<1, \\
\phi(q) & :=f(q, q)=\sum_{n=-\infty}^{\infty} q^{n^{2}}=\frac{(-q:-q)_{\infty}}{(q:-q)_{\infty}} \\
\psi(q) & :=f\left(q, q^{3}\right)=\sum_{n=0}^{\infty} q^{n(n+1) / 2}=\frac{\left(q^{2}: q^{2}\right)_{\infty}}{\left(q: q^{2}\right)_{\infty}}
\end{aligned}
$$

where $(a ; q)_{\infty}:=\prod_{n=0}^{\infty}\left(1-a q^{n}\right)$

$\phi(q)$ and $\psi(q)$ have been used for a long time in the theory of elliptic functions and modular forms. The main purpose of this note is to establish continued fractions for $\phi^{2}(q)$ and $\psi^{2}\left(q^{2}\right)$, and to employ them to derive Jacobi's two-square and two-triangular number theorems.

\section{MAin RESUlts}

THEOREM 1. If $|q|<1$, then

$$
1+\phi^{2}(q)=\frac{a_{1}}{b_{1}+\frac{a_{2}}{b_{2}+}} \frac{a_{3}}{b_{3}}+\cdots,
$$

where

$$
\begin{aligned}
& a_{1}=2, a_{2}=-2 q, a_{3}=\left(q-q^{2}\right)^{2}, \ldots, \\
& a_{n}=(-1)^{n-3} q^{n-3}\left(q+(-1)^{n-2} q^{n-1}\right)^{2}, n \geqslant 3, \text { and } \\
& b_{1}=1, b_{2}=1+q, b_{3}=1+q^{3}, \ldots, b_{n}=1+q^{2 n-3}, n \geqslant 3 .
\end{aligned}
$$


ProOF: From ([2, p. 14, Entry 11]), we have

$$
\frac{(-a)_{\infty}(b)_{\infty}-(a)_{\infty}(-b)_{\infty}}{(-a)_{\infty}(b)_{\infty}+(a)_{\infty}(-b)_{\infty}}=\frac{a-b}{1-q}+\frac{(a-b q)(a q-b)}{1-q^{3}}+\frac{q\left(a-b q^{2}\right)\left(a q^{2}-b\right)}{1-q^{5}}+\cdots
$$

This continued fraction identity may be rewritten as

$$
1+\frac{(-a)_{\infty}(b)_{\infty}}{(a)_{\infty}(-b)_{\infty}}=\frac{2}{1-} \frac{a-b}{1-q}+\frac{(a-b q)(a q-b)}{1-q^{3}}+\frac{q\left(a-b q^{2}\right)\left(a q^{2}-b\right)}{1-q^{5}}+\cdots
$$

Changing $q$ to $-q, a$ to $q$ and $b$ to $-q$ in the above continued fraction identity and using (1.4), we complete the proof.

THEOREM 2. If $|q|<1$, then

$$
2+4 \sum_{n=1}^{\infty} \frac{(-1)^{n(n-1) / 2} q^{n(n+1) / 2}}{1+(-q)^{n}}=\frac{a_{1}}{b_{1}}+\frac{a_{2}}{b_{2}}+\frac{a_{3}}{b_{3}}+\cdots,
$$

where $a_{n}$ and $b_{n}$ are as defined in Theorem 1.

Proof: Let $P_{n} / Q_{n}$ denote the $n$-th convergent of the continued fraction $a_{1} / b_{1}+a_{2} / b_{2}+a_{3} / b_{3}+\cdots$. Then

$$
\begin{aligned}
& Q_{1}=1, Q_{2}=(1-q), Q_{3}=\left(1+q^{3}\right)(1-q)+\left(q-q^{2}\right)^{2}=(1-q)\left(1+q^{2}\right) \text { and } \\
& Q_{n}=b_{n} Q_{n-1}+a_{n} Q_{n-2} .
\end{aligned}
$$

By induction on $n$, we can show that

$$
Q_{n}=\prod_{k=1}^{n-1}\left(1+(-1)^{k} q^{k}\right), n \geqslant 2 .
$$

We have

$$
\begin{aligned}
\frac{P_{n}}{Q_{n}} & =\sum_{k=1}^{n} \frac{(-1)^{k+1} a_{1} a_{2} \ldots a_{k}}{Q_{k} Q_{k-1}} \\
& =\frac{a_{1}}{Q_{1} Q_{0}}-\frac{a_{1} a_{2}}{Q_{2} Q_{1}}+\frac{a_{1} a_{2} a_{3}}{Q_{3} Q_{2}}-\cdots+\frac{(-1)^{n+1} a_{1} a_{2} a_{3} \ldots a_{n}}{Q_{n} Q_{n-1}} \\
& =\frac{2}{1}+\frac{4 q}{1-q}-\frac{4 q^{3}}{1+q^{2}}-\frac{4 q^{6}}{1-q^{3}}+\frac{4 q^{10}}{1+q^{4}}+\cdots+4 \frac{(-1)^{((n-1)(n-2)) / 2} q^{n(n-1) / 2}}{1+(-q)^{n-1}} .
\end{aligned}
$$

Letting $n \rightarrow \infty$, we obtain the required result.

THEOREM 3. Jacobi's two-square theorem.

$$
r_{2}(n)=4\left[d_{1,4}(n)-d_{3,4}(n)\right] .
$$

Proof: From (2.1) and (2.2), we have

$$
\begin{aligned}
\phi^{2}(q) & =1+4 \sum_{n=1}^{\infty} \frac{(-1)^{n(n-1) / 2} q^{n(n+1) / 2}}{1+(-q)^{n}} \\
& =1+4 \sum_{n=1}^{\infty}\left(\frac{q^{4 n-3}}{1-q^{4 n-3}}-\frac{q^{4 n-1}}{1-q^{4 n-1}}\right)
\end{aligned}
$$


on summing by the column-row method. Now, comparing the coefficients of $q^{n}$ on both sides of (2.3), we obtain Jacobi's two-square Theorem.

THEOREM 4. If $|q|<1$, then

$$
\psi^{2}\left(q^{2}\right)=\frac{1}{1-q}+\frac{q(1-q)^{2}}{(1-q)\left(1+q^{2}\right)}+\frac{q\left(1-q^{3}\right)^{2}}{(1-q)\left(1+q^{4}\right)}+\frac{q\left(1-q^{5}\right)^{2}}{(1-q)\left(1+q^{6}\right)}+\cdots
$$

Proof: From ([2, p. 17, Entry 12]), we have

$$
\frac{\left(a^{2} q^{3} ; q^{4}\right)_{\infty}\left(b^{2} q^{3} ; q^{4}\right)_{\infty}}{\left(a^{2} q ; q^{4}\right)_{\infty}\left(b^{2} q ; q^{4}\right)_{\infty}}=\frac{1}{1-a b}+\frac{(a-b q)(b-a q)}{(1-a b)\left(1+q^{2}\right)}+\frac{\left(a-b q^{3}\right)\left(b-a q^{3}\right)}{(1-a b)\left(1+q^{4}\right)}+\cdots
$$

Putting $a=b=\sqrt{q}$ in the above identity and using (1.5), we obtain (2.4).

THEOREM 5. If $|q|<1$, then

$$
\sum_{n=0}^{\infty} \frac{(-1)^{n} q^{n}}{1-q^{2 n+1}}=\frac{1}{1-q}+\frac{q(1-q)^{2}}{(1-q)\left(1+q^{2}\right)}+\frac{q\left(1-q^{3}\right)^{2}}{(1-q)\left(1+q^{4}\right)}+\cdots .
$$

Proof: Let $P_{n} / Q_{n}$ denote the n-th convergent of the continued fraction (2.5). Then

$$
Q_{1}=1-q, Q_{2}=(1-q)^{2}\left(1+q^{2}\right)+q(1-q)^{2}=(1-q)^{2}\left(1+q+q^{2}\right)=(1-q)\left(1-q^{3}\right)
$$

By induction on $n$, one can show that

$$
Q_{n}=(1-q)\left(1-q^{3}\right)\left(1-q^{5}\right) \ldots\left(1-q^{2 n-1}\right) .
$$

Then we have

$$
\begin{aligned}
\frac{P_{n}}{Q_{n}} & =\sum_{k=1}^{n} \frac{(-1)^{k+1} a_{1} a_{2} \ldots a_{k}}{Q_{k} Q_{k-1}} \\
& =\frac{a_{1}}{Q_{1} Q_{0}}-\frac{a_{1} a_{2}}{Q_{2} Q_{1}}+\frac{a_{1} a_{2} a_{3}}{Q_{3} Q_{2}}-\cdots+\frac{(-1)^{n+1} a_{1} a_{2} a_{3} \ldots a_{n}}{Q_{n} Q_{n-1}} \\
& =\frac{1}{1-q}-\frac{q}{1-q^{3}}+\frac{q^{2}}{1-q^{5}}-\cdots+\frac{(-1)^{n-1} q^{n-1}}{1-q^{2 n-1}} .
\end{aligned}
$$

Letting $n \rightarrow \infty$, we complete the proof of Theorem 5 .

THEOREM 6. Jacobi's two-triangular number Theorem.

$$
t_{2}(n)=d_{1}(4 n+1)-d_{3}(4 n+1)
$$

Proof: From (2.4) and (2.5), we have

$$
\psi^{2}\left(q^{2}\right)=\sum_{n=0}^{\infty} \frac{(-1)^{n} q^{n}}{1-q^{2 n+1}}
$$


Changing $q$ to $-q$ in (2.6) and adding the resulting identity to (2.6), we obtain

$$
\begin{aligned}
2 \psi^{2}\left(q^{2}\right) & =\sum_{n=0}^{\infty} \frac{(-1)^{n} q^{n}}{1-q^{2 n+1}}+\sum_{n=0}^{\infty} \frac{q^{n}}{1+q^{2 n+1}} \\
& =2 \sum_{n=0}^{\infty} \frac{q^{2 n}}{1-q^{8 n+2}}-2 \sum_{n=0}^{\infty} \frac{q^{6 n+4}}{1-q^{8 n+6}} .
\end{aligned}
$$

Changing $q$ to $q^{1 / 2}$ in (2.7), we deduce

$$
\begin{aligned}
\psi^{2}(q) & =\sum_{n=0}^{\infty} \frac{q^{n}}{1-q^{4 n+1}}-\sum_{n=0}^{\infty} \frac{q^{3 n+2}}{1-q^{4 n+3}} \\
& =\sum_{n, m=0}^{\infty} q^{4 m n+m+n}-\sum_{n, m=0}^{\infty} q^{4 m n+3 m+3 n+2} \\
& =\sum_{n, m=0}^{\infty} q^{((4 m+1)(4 n+1)-1) / 4}-\sum_{n, m=0}^{\infty} q^{((4 m+3)(4 n+3)-1) / 4} .
\end{aligned}
$$

By comparing the coefficients of $q^{n}$ on both sides of (2.8), we obtain the Jacobi's twotriangular number Theorem.

\section{REFERENCES}

[1] C. Adiga, 'On the representation of an integer as a sum of two or four triangular numbers', Nihonkai Math. J. 3 (1992), 125-131.

[2] C. Adiga, B.C. Berndt, S. Bhargava and G.N. Watson, 'Chapter 16 of Ramanujan's second notebook: Theta-functions and $q$-series', Mem. Am. Math. Soc. 53 (1985).

[3] R. Askey, 'The number of representations of an integer as the sum of two squares', Indian J.Math. 32 (1990), 187-192.

[4] S. Bhargava and C. Adiga, 'Simple proofs of Jacobi's two and four square theorems', Internat. J. Math. Ed. Sci. Tech. 19 (1988), 779-782.

[5] S. Cooper and H.Y. Lam, 'Sums of two, four, six and eight squares and triangular numbers : An elementary approach', Indian J. Math. 44 (2002), 21-40.

[6] J.A. Ewell, 'On sums of triangular numbers and sums of squares', Amer. Math. Monthly 99 (1992), 752-757.

[7] N. Fine, Basic hypergeometric series and applications, Mathematical Surveys and Monograph 27 (Province, R.I., 1988).

[8] M.D. Hirschhorn, 'A simple proof of Jacobi's two-square theorem', Amer. Math. Monthly 92 (1985), 578-580.

Department of Studies in Mathematics

University of Mysore

Manasa Gangotri

Mysore - 570006

India

e-mail: c_adiga@hotmail.com

jhan176@yahoo.com 